\title{
Computed Tomographic Diagnosis of Traumatic Atlanto-occipital Rotatory Luxation and Successful Closed Reduction in a Dog
}

\author{
Gordon Lye ${ }^{1}$ Karl Mathis ${ }^{2}$ Sarah Hill ${ }^{3}$ Ryan Cattin ${ }^{3}$ Angela Hartman ${ }^{1}$ \\ ${ }^{1}$ Department of Radiology, Animal Referral Centre, Auckland, New \\ Zealand \\ 2 Department of Surgery, Animal Referral Centre, Auckland, New \\ Zealand \\ ${ }^{3}$ Department of Internal Medicine, Animal Referral Centre, Auckland, \\ New Zealand \\ VCOT Open 2020;3:e164-e169. \\ Address for correspondence Gordon Lye, BVSc, Department of \\ Radiology, Animal Referral Centre, 224 Albany Highway, Schnapper \\ Rock, Auckland 0632, New Zealand \\ (e-mail: gordonmlye@gmail.com).
}
Abstract
Keywords
- atlanto-occipital luxation
- dog
- cervical vertebrae trauma
- closed reduction

\begin{abstract}
A 12-year-old, female spayed crossbred dog was presented for traumatic injuries sustained after being hit by a car. The dog was non-ambulatory with a right-sided head tilt on presentation, neurological deficits and signs of external trauma centred on the head. A trauma computed tomography was performed which revealed an atlantooccipital luxation with narrowing of the vertebral canal. This was treated successfully via closed reduction and confirmed with a repeat computed tomography scan. The dog recovered well following the atlanto-occipital luxation reduction, and was ambulatory the day after the reduction, and neurological signs resolved within 2 days.
\end{abstract}

\section{Introduction}

A 12-year-old, $33 \mathrm{~kg}$, female spayed crossbred dog presented 2 hours after being hit by a car. On physical examination, the dog had multiple superficial bleeding wounds over the left side of the head. Bilateral epistaxis was present and harsh lung sounds referred from the upper respiratory tract were heard across both lung fields. The dog was able to stand with support but not ambulate. On neurological exam, there were multiple neurological deficits; clinical signs are summarized in - Table 1. The initial findings were indicative of a brain stem lesion. There was no previous history of major illness.

\section{Case Description}

Baseline blood tests were performed and within normal limits. Following stabilization, the dog was sedated with fentanyl (Biomed, Auckland, New Zealand) at $5 \mu \mathrm{g} / \mathrm{kg}$ intravenously (IV) for a computed tomography (CT) of the head and neck. The CT was performed (Somatom go.Up 64-slice CT Scanner,
Siemens Healthineers, Erlangen, Germany), with the patient positioned in sternal recumbency. Iodinated contrast (Omnipaque $300 \mathrm{mg} / \mathrm{mL}$, GE Healthcare Limited, Auckland, New Zealand) was hand-injected IV at a dosage of $600 \mathrm{mg} / \mathrm{kg}$. The images were taken at the following settings: slice thickness, $1 \mathrm{~mm}$; pitch, 0.35 ; rotation duration, 1.0 second tube voltage, $130 \mathrm{kV}$ and soft and sharp algorithms. Reformatted dorsal and sagittal images were then acquired.

An atlanto-occipital luxation (AOL) was identified ( Fig. 1). ${ }^{1}$ The cranial articular processes of the atlas were displaced toward the left, relative to the occipital condyles. The cranial articular processes of the atlas were rostrally displaced and interlocked with the occipital condyles in a luxated position. The luxation was such that there was a persistent rotary displacement locking the head tilted as seen on physical exam. A small periarticular fracture fragment was noted attached to the right cranial articular process of the atlas. This was suspected to be a periarticular fracture shard from the right occipital condyle. A small fracture fragment was identified and associated with the received

July 31,2020

accepted after revision

October 6, 2020
DOI https://doi.org/

10.1055/s-0040-1721031. ISSN 2625-2325. (c) 2020 Georg Thieme Verlag KG
Stuttgart · New York

License terms

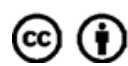


Table 1 Neurological assessment of the dog from presentation before closed reduction, through to discharge

\begin{tabular}{|l|l|l|l|}
\hline Neurological reflexes & Pre-reduction & 1-day post-reduction & 2-days post-reduction \\
\hline Mentation & Dull/depressed & Quiet, responsive & Bright, responsive \\
\hline Head tilt & Present, right sided & None & None \\
\hline Pupillary light-direct & Reduced, bilateral & Present, bilateral & Present, bilateral \\
\hline Pupillary light-consensual & Reduced, bilateral & Present, bilateral & Present, bilateral \\
\hline Pupil size & Symmetrical, normal & Symmetrical, normal & Symmetrical, normal \\
\hline Palpebral & Absent, bilateral & Present, bilateral & Present, bilateral \\
\hline Menace & Absent, bilateral & Present, bilateral & Present, bilateral \\
\hline Gag & Not assessed & Present & Present \\
\hline Forelimb proprioception & Present & Present & Present \\
\hline Hindlimb proprioception & Absent, bilateral & Absent, bilateral & Present, bilateral \\
\hline Withdrawal & Present & Present & Present \\
\hline Gait & Non-ambulatory tetraparesis & Ambulatory paraparesis & Ambulatory mild ataxia \\
\hline
\end{tabular}

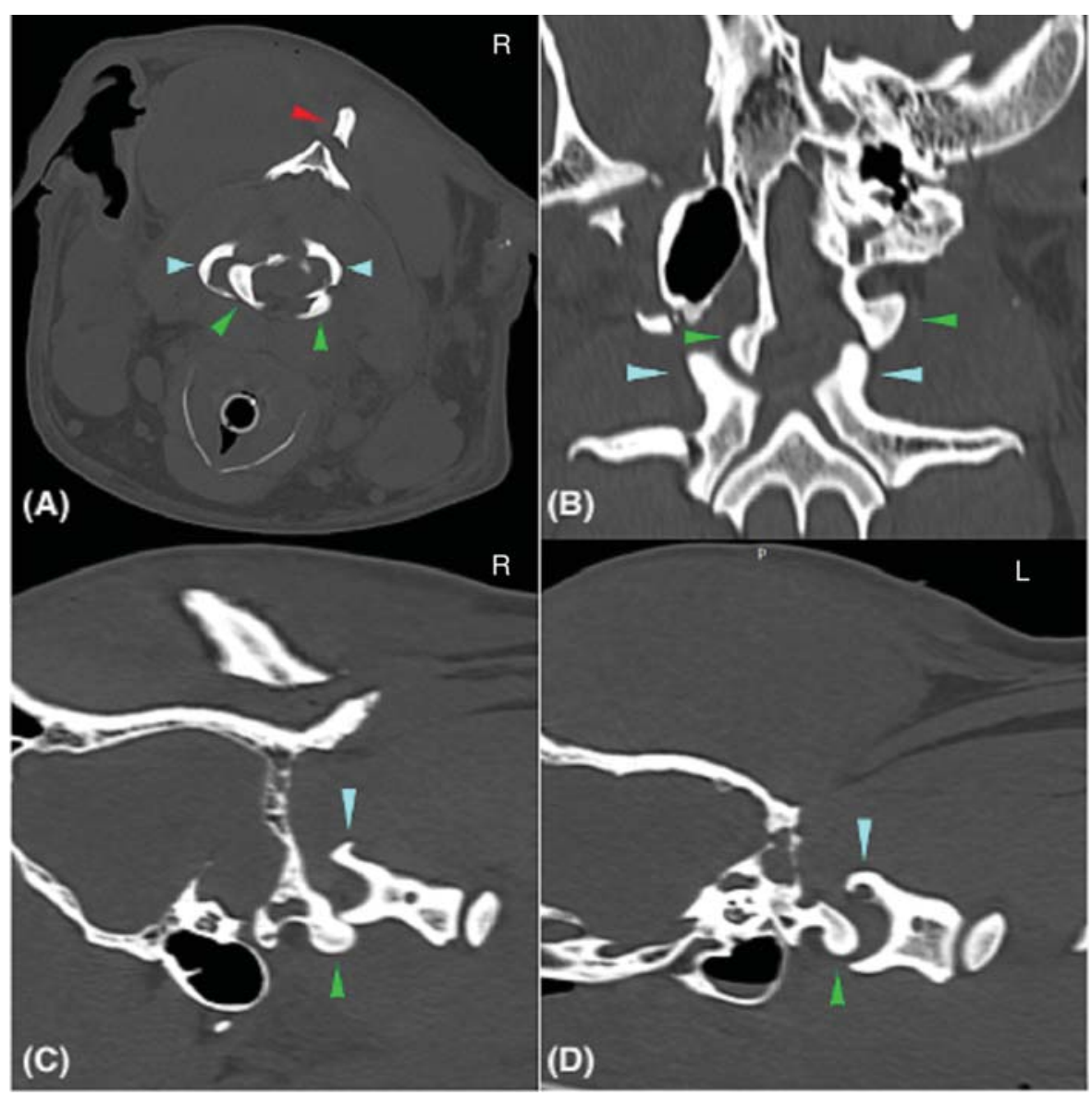

Fig. 1 Cross-sectional computed tomographic images (slice interval $1.0 \mathrm{~mm}$ ) using bone reconstruction algorithm of the dog before closed reduction. The green arrows indicate the occipital condyles and the blue arrows show the atlas. (A) Axial view of atlanto-occipital luxation (AOL). The red arrow shows the external sagittal crest. (B) Dorsal view of AOL. Sagittal view of right (C) and left (D) sides of the AOL.

left transverse process of $\mathrm{C} 1$ at the cranial margin that did not alter its integrity. No other fractures of the occiput or atlas were present. The brain stem was deformed most notably on the right and ventral aspect at the site of luxation. Conforming radiopaque material of $\sim 50$ Hounsfield units was seen surrounding the brain stem at the site of luxation that was most consistent with haemorrhage. The atlantoaxial junction appeared normal. The calvarium was intact with no signs of intracalvarial haemorrhage or increased intracranial pressure identified. 


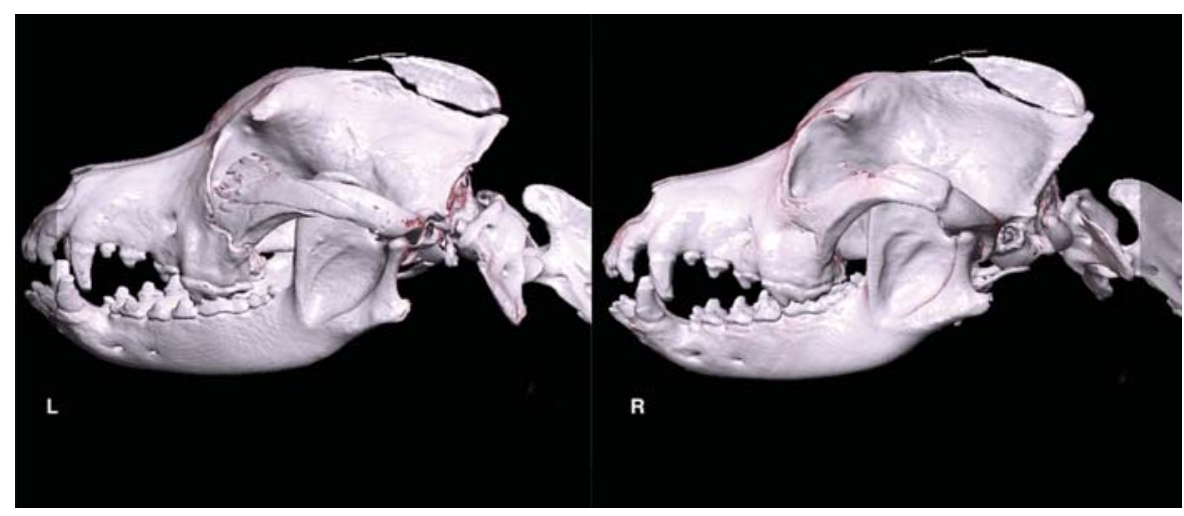

Fig. 2 Three-dimensional reconstructions of the dog's skull in left and right lateral views, illustrating multiple fractures.

There was a comminuted displaced fracture of the left orbit with large bony fragments compressed into the caudal aspect of the left nasal cavity. There was increased fluid opacity within the left orbit thought to be due to haemorrhage and oedema. Mild exophthalmos was seen with the globe intact. A conforming soft tissue opaque material considered consistent with fluid, was seen within the left nasal cavity and the left sphenopalatine sinus. The lateral wall of the left sphenopalatine sinus had a bony fragment compressed axially into its centre, resulting in a large communication between the sinus and the retrobulbar tissues. The olfactory region of the frontal bone was intact. Nondisplaced parasagittal fractures were also found through the right mandibular fossa. A similar parasagittal fracture through the left mandibular fossa of the temporal bone was identified that is mildly displaced. The retroarticular process was also fractured and mildly displaced. A soft tissue opacity was seen within the left bulla and associated external ear canal. A complete, mildly displaced fracture of the sagittal ridge of the calvarium was seen with gas-like opacity compatible with emphysema surrounding the bony fragment. Mildly displaced fractures associated with both zygomatic arches were found, which were worse on the left side (-Fig. 2). No abnormalities were detected in the thorax or abdomen.

Prior to closed reduction, anaesthesia was induced. The dog was maintained on a fentanyl constant rate infusion at $3 \mu \mathrm{g} / \mathrm{kg}$ IV then given a bolus of fentanyl at $5 \mu \mathrm{g} / \mathrm{kg}$ IV and alfaxalone (Jurox, Rutherford, Australia) IV to effect to achieve anaesthetic induction, then intubated with an endotracheal tube and maintained with isoflurane in oxygen.

The AO luxation was treated by closed reduction. The procedure was performed on the CT table to allow for imaging as needed. The dog was positioned in sternal recumbency. A harness was placed onto the dog and secured caudally to the end of the CT table with a ratchet strap. Securing the head was complicated by the zygomatic fractures and lack of undamaged attachment points on the skull. Therefore, a custom harness was designed with strapping tape to attach to the maxillary canine teeth. A second ratchet strap connected this tape harness to a stationary pole beyond the CT gantry. Tightening of the cranial ratchet strap allowed for carefully controlled tension to be applied to allow a slow distraction of the occipital condyles from the interlocked cranial articular facets of the atlas. This set-up is illustrated in -Fig. 3. Careful distraction force was applied using the cranial ratchet straps to distract the head from the body, parallel to the plane of the cervical spine. An assistant manually stabilized the atlas. Once the head was cranially distracted to allow clearance of the occipital condyles from the interlocked atlas, then the head was flexed slightly to the left and carefully rotated clockwise to lift the right occipital condyle over the rim of the articular fovea, thus reducing the atlanto-occipital joint. The occipital condyle was reduced back into apposition with the cranial articular fovea with a slight pop felt. (-Figs. 4 and 5). This process is shown in - Video 1.

\section{Video 1}

Actual closed reduction procedure. Online content including video sequences viewable at: https://www. thieme-connect.com/products/ejournals/html/ 10.1055/s-0040-1721031.

Confirmation of the AO joint reduction was achieved by a repeat CT scan as seen in - Fig. 4. Post-reduction palpation and gentle motion of the craniovertebral junction suggested normal position and function of the articulation of the AO joint.

Recovery from anaesthesia was uneventful, with neurological status improving by the next day (-Table 1). After 2 days of cage rest, the dog was ambulatory and discharged from hospital. The owners were advised to use a harness for restricted activity and short walks. Four weeks after the closed reduction, the dog presented in the clinic for reexamination and was found to be neurologically normal. The dog was ambulatory, bright and responsive. The owners reported that the dog was clinically normal at home. - Video $\mathbf{2}$ shows the dog walking well when it presented for a revisit 4 weeks after discharge. 


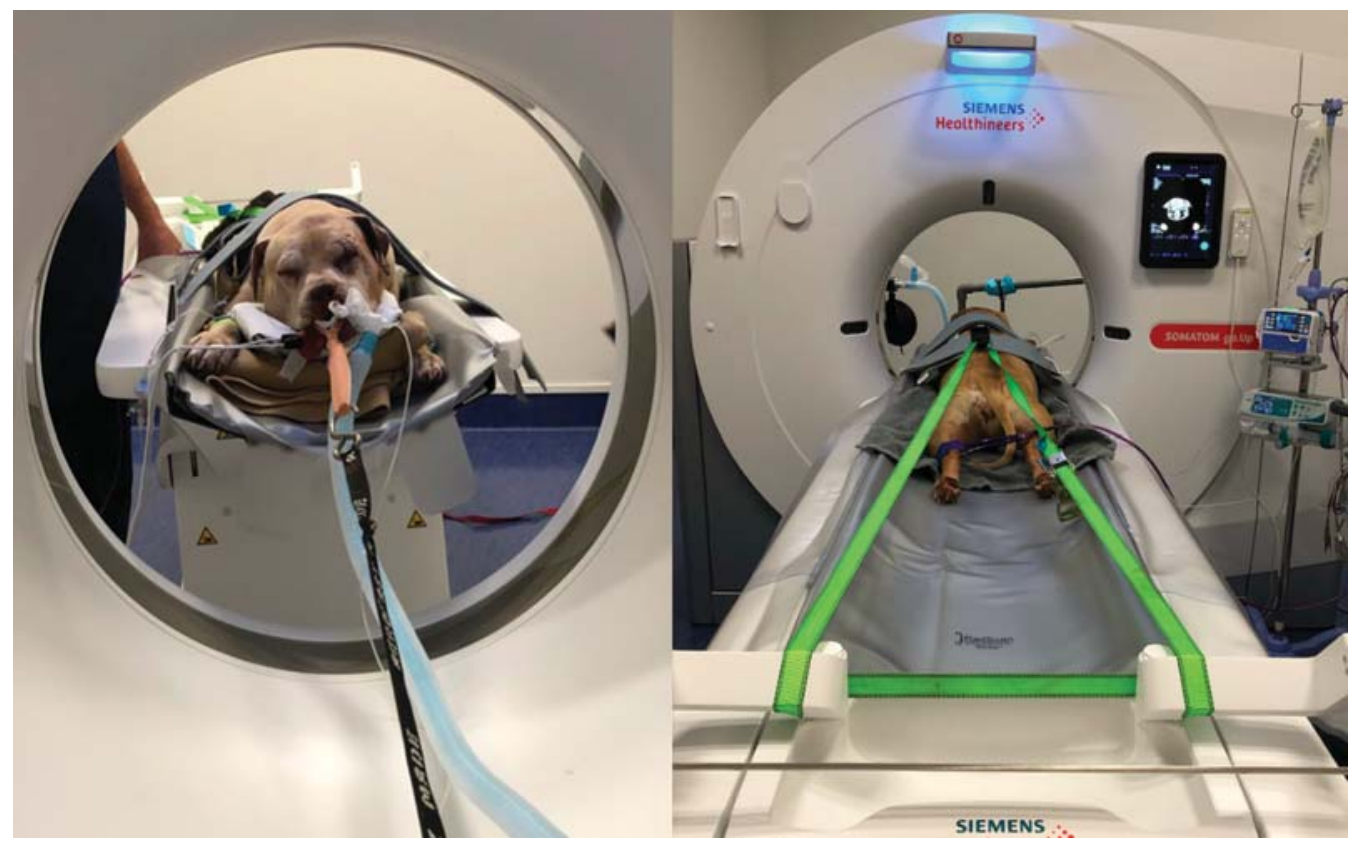

Fig. 3 Photographs demonstrating the positioning and securing of the patient for the closed reduction, with the craniocaudal view on the left and the caudocranial view on the right.

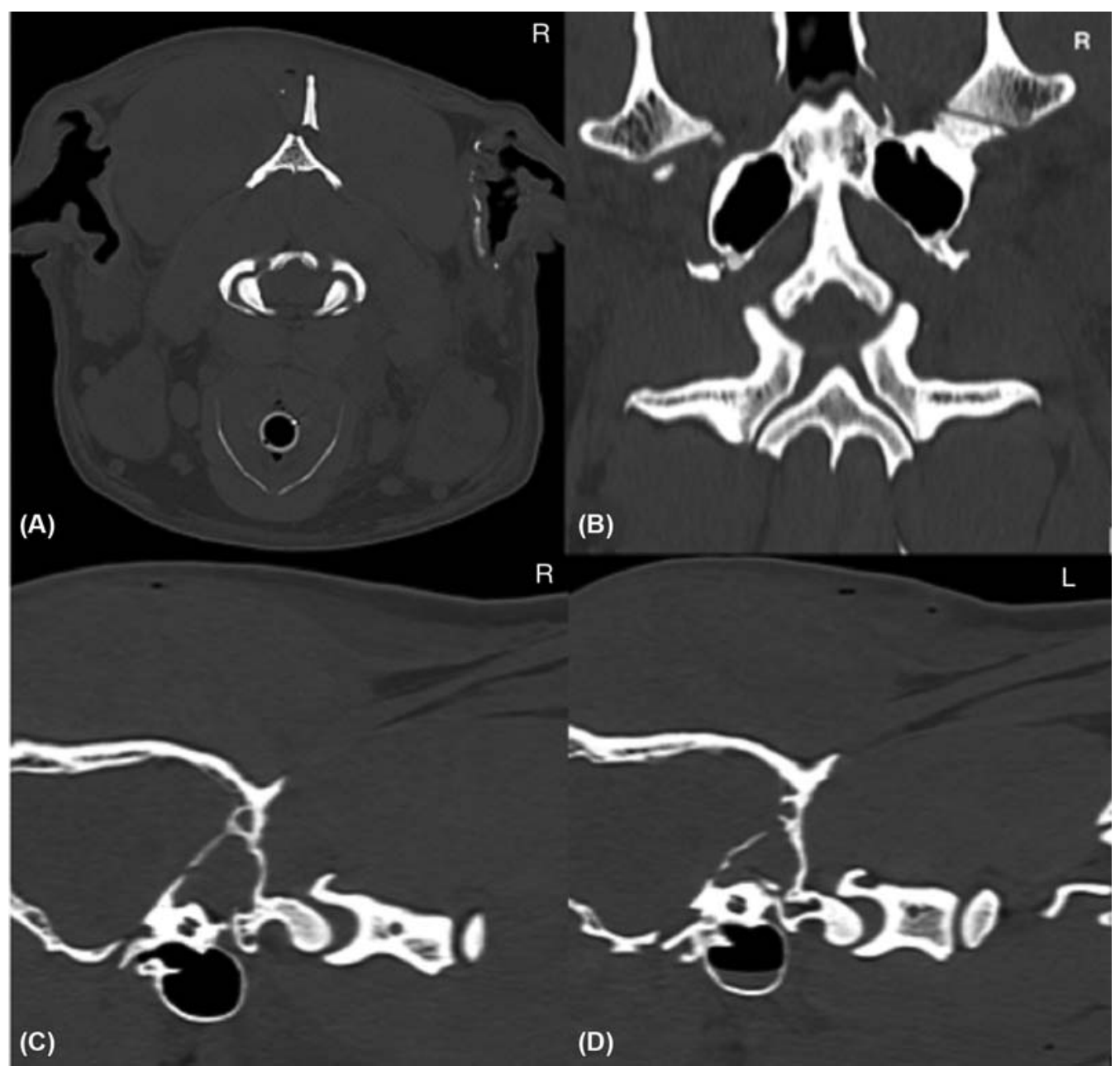

Fig. 4 Cross-sectional computed tomographic images (slice interval $1.0 \mathrm{~mm}$ ) using bone reconstruction algorithm of the dog after successful closed reduction of the atlanto-occipital luxation (AOL). The foramen magnum now aligns with the vertebral canal. (A) Axial view of AO joint. The occipital condyles now sit within the cranial articular fovea of the atlas (B) Coronal view of AO joint. Sagittal view of right (C) and left (D) sides of the AO joint. 


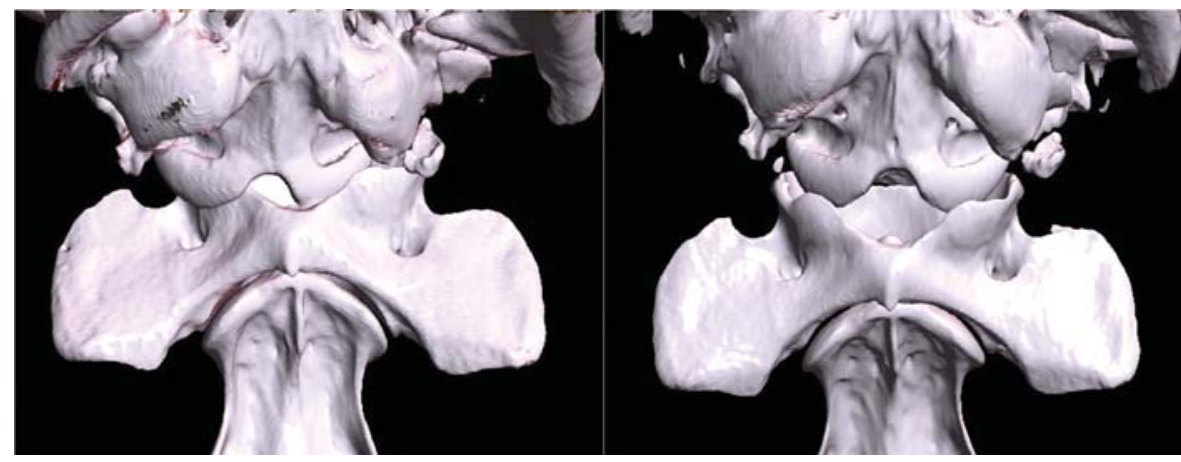

Fig. 5 Three-dimensional reconstructions of the atlanto-occipital luxation pre- and post-reduction (left and right respectively), as seen from the ventral aspect.

\section{Video 2}

The dog walking well when it presented for a revisit 4 weeks after discharge. Online content including video sequences viewable at: https://www.thiemeconnect.com/products/ejournals/html/10.1055/s0040-1721031.

\section{Discussion}

Traumatic luxations of the $\mathrm{AO}$ joint are rarely reported in the veterinary literature. A review of publications found 11 cases (nine canines, one feline and one alpaca) of AOL that survived treatment. ${ }^{2-7}$ Out of those cases, five dogs were treated surgically ${ }^{7}$ and the remaining animals were managed conservatively. $^{2-6}$ This condition is reported to have a high mortality rate in people, ${ }^{1,8}$ so is possible that this injury in dogs is more common than reported with animals perhaps not surviving long enough to be diagnosed with AOL.

Atlanto-occipital luxations in humans occur mainly as ligamentous injuries due to high-speed trauma such as motor vehicle accidents or falls from heights. ${ }^{1,8}$ There are different subtypes of AOLs in people, ${ }^{9,10}$ including atlantooccipital rotatory luxation (AORL). ${ }^{10}$ The AORL is best diagnosed by CT imaging, showing the rotation of both occipital condyles in relation to the atlas. ${ }^{10}$ This description appears to fit best with the displacement seen on CT in the dog of this report. Given the low number of cases reported, no subclassification system exists in the veterinary literature, and no detailed description of the type of dislocation is available in the previous reports.

The AORL in humans has been noted to carry a better clinical outcome than non-rotatory AOL. ${ }^{5,11}$ Treatment in such cases typically involves either surgical reduction and stabilization or stabilization with bracing and splinting. Subclassification for AOLs in dogs may also be appropriate as there may be prognostic and treatment implications as in people; however, more data would be needed to confirm this.

Survival after closed reduction in traumatic AOL has been reported in three dogs, one cat and one alpaca. ${ }^{2,4-6}$
All three dogs had a bilateral dislocation of the occipital condyles, and one of them had an avulsion fracture between the atlas and occiput but without displacement. ${ }^{2}$ In each of these cases, closed reduction was performed by applying traction to the $\mathrm{AO}$ joint and by reducing the luxation by rotation force. Sufficient stability seems to be achieved long-term by the formation of fibrous scar tissue as healing response to injury of the ligaments around the AO joint. ${ }^{5,12}$

This case report highlights the importance of CT imaging in the diagnosis of AOL as well as $\mathrm{CT}$ as a guiding imaging tool for conservative treatment. The spatial orientation of the luxation and the degree of overriding displacement was readily recognized and destabilizing articular fractures could be excluded. With the CT results, the degree of fractures of the caudal skull could be clearly identified so that a stable bony distraction column could be precisely planned. Using the ratchet straps aided in distraction, which improved control during closed reduction. With greater control and understanding of the situation, the $\mathrm{CT}$ images substantially improved 3-dimensional orientation, and thus the closed reduction was achieved relatively quickly. An immediate post-reposition scan confirmed successful reduction. Immediately rescanning with $\mathrm{CT}$ allowed confirmation of successful reduction.

This report shows that closed reduction is a viable option to successfully treat AOL in the dog. Being non-invasive, the described method is relatively quick and avoids additional injury to the surrounding soft tissue structures, as compared to surgical reduction.

\section{Authors' Contributions}

G.L. was responsible for drafting, editing and compiling the manuscript, and was involved with the case management and assisted with closed reduction. K.M. was responsible for editing the manuscript, literature review and engineering the set-up for and performing the closed reduction. S.H. was responsible for editing the manuscript as well as case management and literature review. R.C. was involved with editing the manuscript and literature review. A.H. was involved with the diagnosis and interpretation of the computed tomography as well as editing the manuscript and literature review. 


\section{Conflict of Interest}

None declared.

\section{References}

1 Hall GC, Kinsman MJ, Nazar RG, et al. Atlanto-occipital dislocation. World J Orthop 2015;6(02):236-243

2 Greenwood KM, Oliver JE Jr. Traumatic atlanto-occipital dislocation in two dogs. J Am Vet Med Assoc 1978;173(10):1324-1327

3 Crane SW. Surgical management of traumatic atlanto-occipital instability in a dog. Vet Surg 1978;7(02):39-42

4 Lappin MR, Dow S. Traumatic atlanto-occipital luxation in a cat. Vet Surg 1983;12(01):30-32

5 Steffen F, Flueckiger M, Montavon PM. Traumatic atlanto-occipital luxation in a dog: associated hypoglossal nerve deficits and use of 3-dimensional computed tomography. Vet Surg 2003;32(05): 411-415

6 Baitchman EJ, Aiken SW, Calle PP. Successful treatment of atlantooccipital luxation in an alpaca (Lama pacos). J Zoo Wildl Med 2006;37(01):71-74
7 Dolera M, Malfassi L, Bianchi C, et al. Zygomatic arch-atlas wing stabilization in 5 dogs with atlanto-occipital dislocation. J Vet Med Sci 2016;78(06):963-970

8 Labler L, Eid K, Platz A, Trentz O, Kossmann T. Atlanto-occipital dislocation: four case reports of survival in adults and review of the literature. Eur Spine J 2004;13(02):172-180

9 Traynelis VC, Marano GD, Dunker RO, Kaufman HH. Traumatic atlanto-occipital dislocation. Case report. J Neurosurg 1986;65 (06):863-870

10 Robles LA, Mundis GM, Cuevas-Solórzano A. Atlanto-occipital rotatory dislocation: a case report and systematic review. World Neurosurg 2018;110:106-114

11 Dickman CA, Papadopoulos SM, Sonntag VK, Spetzler RF, Rekate HL, Drabier J. Traumatic occipitoatlantal dislocations. J Spinal Disord 1993;6(04):300-313

12 Watson AG, Evans HE, de Lahunta A. Gross morphology of the composite occipito-atlas-axis joint cavity in the dog. Anat Histol Embryol 1986;15(02):139-146 